\title{
Characteristics of pediatric adverse drug reaction reports in the Japanese Adverse Drug Event Report Database
}

\author{
Aoi Noda ${ }^{1,2,3}$, Takamasa Sakai ${ }^{4}$, Taku Obara ${ }^{1,2,3^{*}}$, Makoto Miyazaki ${ }^{5}$, Masami Tsuchiya ${ }^{5,6}$, Gen Oyanagi ${ }^{3}$, \\ Yuriko Murai ${ }^{7}$ and Nariyasu Mano ${ }^{3,5}$
}

\begin{abstract}
Background: There are no reports on investigations of the characteristics of adverse drug reaction (ADR) reports for pediatric patients in the Japanese Adverse Drug Event Report database (JADER) and the utility of database for drug safety surveillance in these patients.

Method: We aimed to evaluate ADR reports for pediatric patients in the JADER. We used spontaneous ADR reports included in the JADER since April 1, 2004, to December 31, 2017, which was downloaded in April 2018. In a total of 504,407 ADR reports, the number of spontaneous reports was 386,400 (76.6\%), in which 37,534 (7.4\%) were unknown age reports. After extraction of 27,800 ADR reports for children aged $<10$ and 10-19years, we excepted for ADR reports associated with a vaccine $(n=6355)$ and no-suspected drug reports $(n=86)$. A total of 21,359 (4.2\%) reports were finally included in this analysis.

Results: More than half of the ADR reports were for children aged < 10 years. Approximately $30 \%$ of ADR reports had multiple suspected drugs, which did not differ by age. The percentages of fatal outcomes of ADRs among patients aged $<10$ and 10-19 years were 4.7 and 3.9\%, respectively. The most frequently reported drug, reaction, and drug-reaction pair were oseltamivir, abnormal behavior, and oseltamivir and abnormal behavior, respectively.

Conclusion: We clarified the characteristics of ADR reports for Japanese children by using the JADER. ADR report databases, especially those for pediatric patients, are valuable pharmacovigilance tools in Japan and other countries. Therefore, a proper understanding of the characteristics of the ADR reports in the JADER is important. Additionally, potential signals for ADRs in pediatric patients should be monitored continuously and carefully.
\end{abstract}

Keywords: Adverse drug reaction, Pediatric patients, Children, The JADER, Spontaneous reports, Drug safety, Pharmacovigilance, Signal detection

\footnotetext{
* Correspondence: obara-t@hosp.tohoku.ac.jp

'Division of Preventive Medicine and Epidemiology, Tohoku University

Tohoku Medical Megabank Organization, Sendai, Miyagi, Japan

${ }^{2}$ Tohoku University Graduate School of Medicine, Sendai, Miyagi, Japan

Full list of author information is available at the end of the article
}

(c) The Author(s). 2020 Open Access This article is licensed under a Creative Commons Attribution 4.0 International License, which permits use, sharing, adaptation, distribution and reproduction in any medium or format, as long as you give appropriate credit to the original author(s) and the source, provide a link to the Creative Commons licence, and indicate if changes were made. The images or other third party material in this article are included in the article's Creative Commons licence, unless indicated otherwise in a credit line to the material. If material is not included in the article's Creative Commons licence and your intended use is not permitted by statutory regulation or exceeds the permitted use, you will need to obtain permission directly from the copyright holder. To view a copy of this licence, visit http://creativecommons.org/licenses/by/4.0/. The Creative Commons Public Domain Dedication waiver (http://creativecommons.org/publicdomain/zero/1.0/) applies to the data made available in this article, unless otherwise stated in a credit line to the data. 


\section{Background}

Spontaneous reporting systems for adverse drug reactions (ADRs) are essential for post-marketing drug safety surveillance [1]. Such systems have been widely used for many drug safety studies. Because nationally compiled data, especially pediatric patient data, may be limited, the Global Research in Pediatrics-Network of Excellence (GRiP) network aims to facilitate the development and safe use of medicine in children and is valuable for examining drug safety [2]. The GRiP project describes the characteristics of individual case safety reports (ICSRs) as reported in a spontaneous reporting database operated by Food and Drug Administration in the United States [1]. Although, in general, a spontaneous ADR reporting database has some limitations such as a lack of denominator of users, an understanding of the structure and scope of the datasets and the respective strengths and limitations of such a database is essential for correct use and interpretation. An understanding of the characteristics of a database is the first and important step for evaluating and developing new methodologies for pharmacovigilance or drug safety [1]. Several retrospective studies of database for ADR reports have clarified their characteristics and availability for use as a database of drug safety surveillance among children in other countries, including the United States [3], Sweden [4], the United Kingdom [5], France [6], Malaysia [7], Spain [8], and Nigeria [9].

The regulatory authority in Japan began collecting ADR reports after the enactment of a law in 1961. Information on serious ADRs from individual cases and study reports from industries, direct voluntary reports from medical institutions, study results from treatment outcome studies, and post-marketing clinical trials has been accumulated since the enactment of the law. Post-2004 ADR reports have been compiled in the Japanese Adverse Drug Event Report database (JADER), which includes some items from ICSRs, such as patient demographic information, drug information, adverse events, and primary illness. This information became available for free download to anyone from the Pharmaceutical and Medical Devices Agency (PMDA) website since 2012 (https://www.info. pmda.go.jp/fukusayoudb/CsvDownload.jsp). This pharmacovigilance database provides a general picture of ADRs and suggests the relative plausibility using quantitative signal detection methodologies. However, there are no studies investigating the characteristics and utility of the JADER as a resource for drug safety surveillance in pediatric patients. Hence, in this study, we studied ADR reporting for pediatric patients in the JADER with an aim to elucidate the characteristics of the ADR reports therein in pediatric patients.

\section{Methods}

We used spontaneous ADR reports included in the JADER since April 1, 2004, to December 31, 2017, which was downloaded in April 2018. The ADR reports are checked and evaluated whether the ADR report is serious or not before being registered in the JADER by the PMDA, and the JADER in principle comprises serious ADR reports selected by the PMDA. A single ADR report often includes multiple ADRs, which can include non-serious events such as pyrexia and rash. The PMDA recommend companies and healthcare professionals to report ADRs through a system called the Drugs and Medical Devices Safety Information Reporting System, even if the causal relationship between medication use and ADR was unclear. As for patients, the Direct Patient Reporting System for ADR, in which patients and consumers can report ADRs directly to the PMDA, was tentatively started from 2012 as a pilot program and a full-scale operation of the system was started on March 26, 2019. However, the JADER has not included the reports from this system yet. The JADER consists of four tables: (1) patient demographic information (2) drug information (3) adverse events, and (4) primary disease. We extracted spontaneous reports from companies and healthcare facilities. Spontaneous reports were defined as ADR reports derived from unsolicited sources in the International Conference on Harmonization of Technical Requirements for Registration of Pharmaceuticals for Human Use guideline E2B, which included direct reports from healthcare facilities or companies, ADR reports from abstracts, literature, Internet, etc. Because a different system exists for the reporting of adverse reactions due to vaccines, vaccine reports were excluded. The adverse reaction and primary disease fields in the JADER are described by using the Medical Dictionary for Regulatory Activities/Japanese version (MedDRA $\% / \mathrm{J}$ ) and were coded as preferred terms (PTs). We used Med$\mathrm{DRA}^{\oplus / J}$ Version 21.0 in the present study. The information included patient details (age and sex), type of report sender, reporters, suspected drugs, outcomes from ADR reports, and ADRs coded according to PTs. Age, sex, type of report sender (company or healthcare facility), reporters (doctor, pharmacist, healthcare professional, consumer, or lawyer), number of suspected drugs per ADR report, outcomes from ADR reports (cured, recovering, did not recover, recovering with sequelae, death, or unexplained) were collected. As for suspected drugs, we collected both International Nonproprietary Name (INN) and brand name and used INN to treat drugs with the same ingredients as the same drugs for analysis. Since the JADER only included age information as a categorical 
variable, we extracted ADR reports for children aged $<10$ and 10-19years. The 10 most frequently reported drugs, reactions, and drug-reaction pairs were determined according to age $(<10$ years and 10-19 years). Time trends for the number of reports and the frequently reported drug, reaction, and drugreaction pair were also determined. Adverse events were considered serious when they resulted in death, were life threatening, required hospitalization or prolongation of existing hospitalization, resulted in persistent or significant disability or incapacity, were congenital abnormalities or birth defects or were any other medically significant events.

\section{Results}

\section{Characteristics of the reports in the JADER}

A total of 504,407 ADR reports from April 2004 to December 2017 were downloaded from the JADER in April 2018. Of these, the number of spontaneous reports was 386,400 (76.6\%), in which 37,534 (7.4\%) were unknown age reports. After extraction of 27,800 ADR reports for children aged $<10$ and 10-19 years, we excepted for ADR reports associated with a vaccine $(n=6355)$ and no-suspected drug reports $(n=86)$. A total of 21,359 (4.2\%) reports were finally included in this analysis. More than half of the ADR reports pertained to children aged $<10$ years (Table 1 ). In the ADR notifications, the distribution of patients by sex was $53.5 \%$ boy and $40.5 \%$ girl for patients aged < 10 years and $51.3 \%$ boy and $46.5 \%$ girl for patients aged 10-19 years. Regardless of age, most of the reports in the JADER were sent by companies and $>70 \%$ were sent by doctors. Figure 1 shows the steadily increasing trend in the number of ADR reports. Approximately 30\% of ADR reports had multiple suspected drugs, which did not differ by age (Table 1). For patients aged $<10$ years, there were 11,786 ADR reports in total, of which $552(4.7 \%)$ were fatal ADR reports with death reported as an outcome. For patients aged 10-19 years, there were 9573 ADR reports in total, of which 369 (3.9\%) were fatal ADR reports with death as an outcome. The proportion of fatal ADR reports was higher when ADR reports had multiple suspected drugs (Table 1).

\section{Outcomes associated with ADR reports}

For patients aged $<10$ years, in the 11,786 reports, a total of 18,309 ADRs were reported. The percentages of patients who were cured, recovering, and recovering with sequelae were $43.1 \%(n=7898), 23.4 \%(n=4288)$, and $1.8 \%(n=338)$, respectively; $5.4 \%(n=993)$ of the patients did not recover. The percentage of fatal outcomes was $4.4 \%(n=803)$. For patients aged $10-19$ years, in the 9573 reports, a total of 15,419 ADRs were reported. The percentages of patients who were cured, recovering, and
Table 1 Characteristics of ADR reports according to age group

\begin{tabular}{|c|c|c|}
\hline & \multicolumn{2}{|l|}{ Age group } \\
\hline & $\begin{array}{l}<10 \text { years } \\
n=11,786(55.2 \%)\end{array}$ & $\begin{array}{l}10-19 \text { years } \\
n=9573(44.8 \%)\end{array}$ \\
\hline \multicolumn{3}{|l|}{ Sex } \\
\hline Boy, n (\%) & $6305(53.5)$ & $4910(51.3)$ \\
\hline Girl, n (\%) & $4777(40.5)$ & $4449(46.5)$ \\
\hline Unexplained, n (\%) & $704(6.0)$ & $214(2.2)$ \\
\hline \multicolumn{3}{|l|}{ Report source } \\
\hline Company, n (\%) & $11,652(98.9)$ & $9430(98.5)$ \\
\hline Healthcare facility, n (\%) & $134(1.1)$ & $143(1.5)$ \\
\hline \multicolumn{3}{|l|}{ Reporter } \\
\hline Doctor, n (\%) & $10,002(78.4)$ & $7901(74.8)$ \\
\hline Pharmacist, n (\%) & $1218(9.5)$ & $1208(11.4)$ \\
\hline Healthcare professional, n (\%) & $430(3.4)$ & $480(4.5)$ \\
\hline Consumer, n (\%) & $449(3.5)$ & $501(4.7)$ \\
\hline Lawyer, n (\%) & $2(0.0)$ & $0(0.0)$ \\
\hline Unexplained, n (\%) & $656(5.1)$ & $472(4.5)$ \\
\hline Total, n (\%) & $12,757(100)$ & $10,562(100)$ \\
\hline
\end{tabular}

Number of suspected drugs per ADR report

$\begin{array}{lll}1, n(\%) & 8248(70.0) & 6679(69.8) \\ 2, n(\%) & 1824(15.5) & 1449(15.1) \\ 3, n(\%) & 798(6.7) & 634(6.6) \\ 4, n(\%) & 394(3.3) & 322(3.4) \\ 5, n(\%) & 234(2.0) & 165(1.7) \\ 6, n(\%) & 100(0.8) & 131(1.4) \\ 7, n(\%) & 71(0.6) & 68(0.7) \\ 8, n(\%) & 52(0.4) & 33(0.3) \\ 9, n(\%) & 24(0.2) & 31(0.3) \\ \geq 10, n(\%) & 41(0.3) & 61(0.6)\end{array}$

Number of suspected drugs per fatal ADR report (n)

\begin{tabular}{lll}
$1, n(\%)$ & $328(4.0)$ & $215(3.2)$ \\
$2, n(\%)$ & $93(5.1)$ & $61(4.2)$ \\
$3, n(\%)$ & $57(7.1)$ & $38(6.0)$ \\
$4, n(\%)$ & $28(7.1)$ & $16(5.0)$ \\
$\geq 5, n(\%)$ & $46(8.8)$ & $39(8.0)$ \\
Total, n (\%) & $552(4.7)$ & $369(3.9)$ \\
\hline
\end{tabular}

recovering with sequelae were $44.1 \%(n=6805), 22.6 \%$ $(n=3492)$, and $1.1 \%(n=162)$, respectively; $4.4 \%(n=$ 684 ) of the patients did not recover. The percentage of fatal outcomes was $3.3 \%(n=512)$.

\section{Frequently reported drugs}

The most frequently reported drugs in ADR reports for patients aged $<10$ and $10-19$ years were oseltamivir $(2.8 \%)$ and zanamivir (2.7\%), respectively. There were 


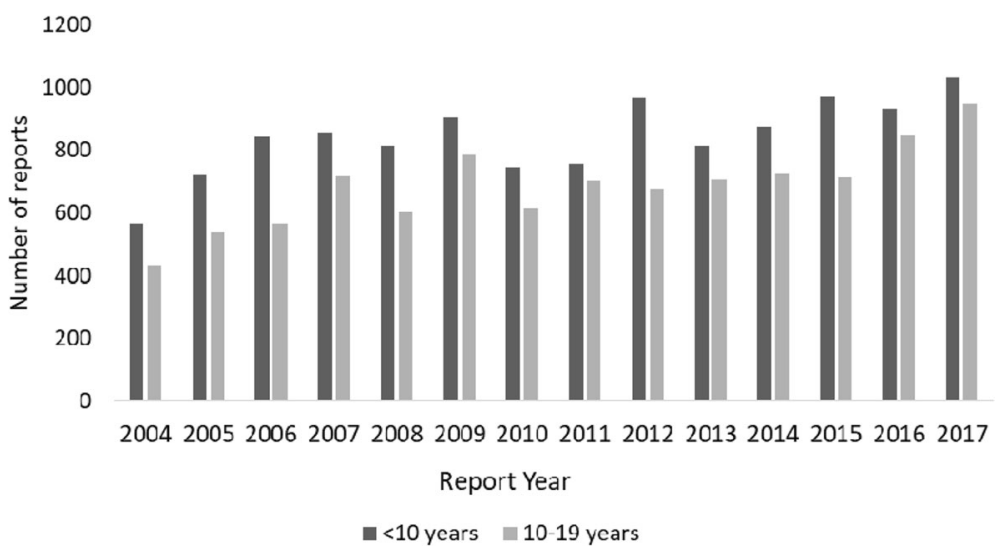

Fig. 1 Annual ADR reports pertaining to children in Japan for 2004-2017 according to age group. ADR: adverse drug reaction

many ADR reports associated with immunosuppressants such as tacrolimus, cyclosporine, and prednisolone, which did not differ according to age. (Table 2). There were many ADR reports for oseltamivir from 2004 to 2008, especially in 2007 for patients aged < 10 years. In 2009, there were many ADR reports for zanamivir for patients aged 10-19 years (Fig. 2). Among 1128 and 764 reported drugs of 552 and 369 fatal ADR reports for patients aged $<10$ and $10-19$ years, the most frequently

Table $\mathbf{2}$ Ten most frequently reported drugs according to age group

\begin{tabular}{ll}
\hline a. < 10 years $(\mathrm{n}=19,829)$ & \\
\hline Oseltamivir, $\mathrm{n}(\%)$ & $540(2.8)$ \\
Cyclosporine, $\mathrm{n}(\%)$ & $413(2.1)$ \\
Tacrolimus, $\mathrm{n}(\%)$ & $387(2.0)$ \\
Prednisolone, $\mathrm{n}(\%)$ & $343(1.8)$ \\
Acetaminophen, $\mathrm{n}(\%)$ & $341(1.8)$ \\
Sodium valproate, $\mathrm{n}(\%)$ & $313(1.6)$ \\
Carbamazepine, n (\%) & $304(1.6)$ \\
Methotrexate, n (\%) & $258(1.3)$ \\
Cefditoren pivoxil, n (\%) & $257(1.3)$ \\
Ceftriaxone sodium, n (\%) & $249(1.3)$ \\
b. 10-19years (n=16,552) & \\
Zanamivir, n (\%) & $440(2.7)$ \\
Prednisolone, n (\%) & $439(2.7)$ \\
Cyclosporine, n (\%) & $384(2.3)$ \\
Tacrolimus, n (\%) & $375(2.3)$ \\
Methotrexate, n (\%) & $371(2.2)$ \\
Carbamazepine, n (\%) & $370(2.2)$ \\
Acetaminophen, n (\%) & $303(1.8)$ \\
Oseltamivir, n (\%) & $295(1.8)$ \\
L-Asparaginase, n (\%) & $244(1.5)$ \\
Cyclophosphamide, n (\%) & $236(1.4)$ \\
\hline
\end{tabular}

reported drugs were etoposide (3.6\%) and tacrolimus $(5.1 \%)$, respectively.

\section{Frequently reported reactions}

For patients aged $<10$ and $10-19$ years, the most frequently reported reactions were seizure $(2.2 \%)$ and abnormal behavior $(2.8 \%)$, respectively (Table 3 ). The time trend for frequently reported reactions was abnormal behavior from 2007 to 2009, and it did not differ by age (Fig. 3). Among 1095 and 768 reported drugs of 552 and 369 fatal ADR reports for patients aged $<10$ and $10-19$ years, the most frequently reported reactions were" death" (3.0\%) and sepsis (3.4\%), respectively.

\section{Frequently reported drug-reaction pairs}

The most frequently reported drug-reaction pairs were "oseltamivir and abnormal behavior" (0.8\%) and "zanamivir and abnormal behavior" (0.8\%) in patients aged < 10 and 10-19 years, respectively (Table 4). The time trends for frequently reported drug-reaction pairs were "oseltamivir and abnormal behavior" in 2007 and "zanamivir and abnormal behavior" in 2009, which did not differ by age (Fig. 4). Among 2363 and 1852 reported drug-reaction pairs of 552 and 369 fatal ADR reports for patients aged $<10$ and $10-19$ years, the most frequently reported drug-reaction pairs were "etoposide and acute respiratory distress syndrome" $(0.3 \%)$ and "bortezomib and neutropenia" $(0.4 \%)$, respectively.

\section{Discussion}

In this study, the number of ADR reports from reporters other than companies, especially pharmacists are low. Although most Japanese hospital pharmacists sufficiently understood the spontaneous ADR reporting system, they also had some barriers to report the ADR such as what kind of ADR to be reported [10]. Additionally, companies are required strictly to report all ADRs within the reporting deadline, differently from healthcare facilities. 
a.

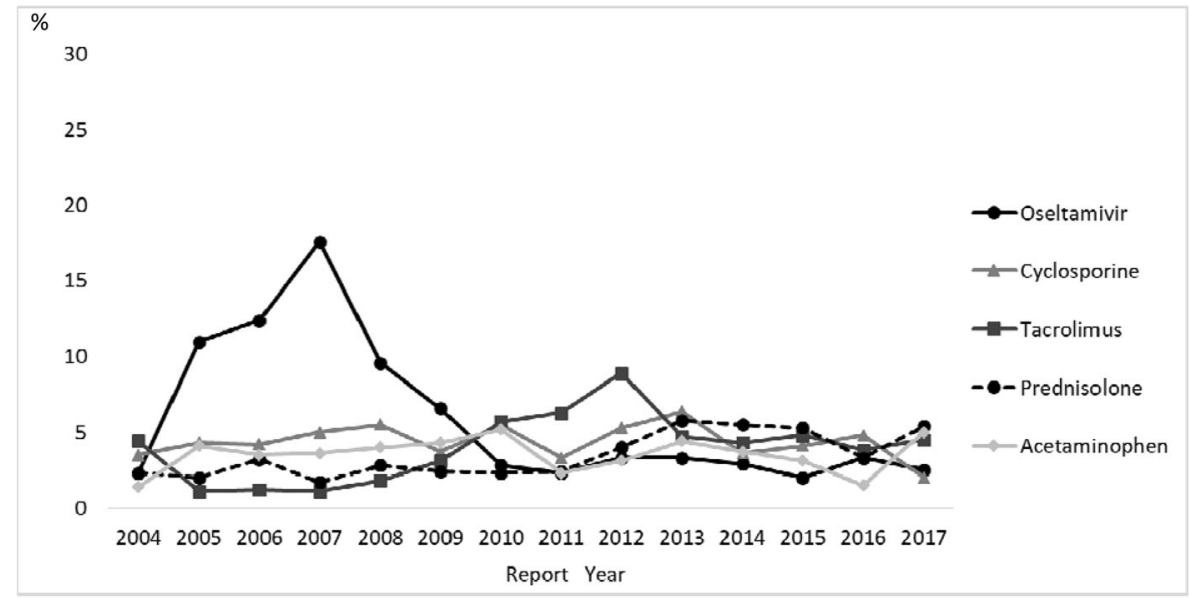

b.

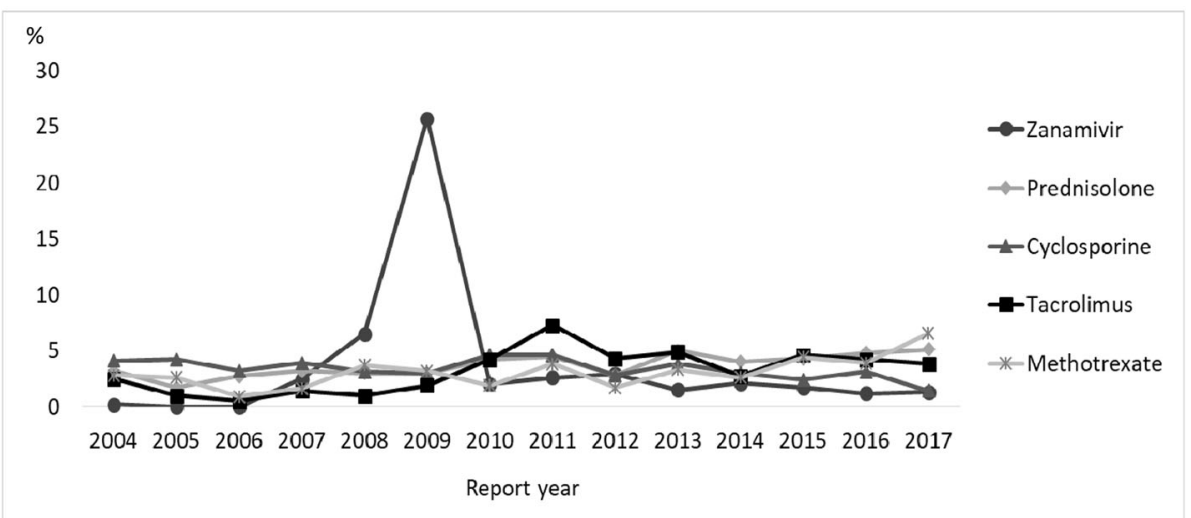

Fig. 2 Time trend for the five most frequently reported drugs according to age group. a children aged $<10$ years; $\mathbf{b}$ children aged 10-19years

Therefore, compared to the healthcare facility, the number of ADR reports from the company might be relatively high.

In Japan, 3.3-4.4\% of pediatric ADR reports during the study period were related to fatal cases, which was higher than the corresponding percentage in other countries $(0.37 \%$ in the UK, $0.24 \%$ in Malaysia, and $0.49 \%$ in Spain) $[7,8,11]$. One of the reasons for the higher percentage of fatal cases was that the JADER is a spontaneous ADR database that in principle comprises serious ADR reports selected by the PMDA and databases in other countries included non-serious ADRs. Therefore, the percentage of fatal cases may reflect differences in the use of medicines and attitudes toward reporting in different countries [12].

Fatal ADR reports are the cases where outcomes are described as death and tend to be reported more positively because of their importance and difficulty in understanding. Our study found that the percentage of fatal ADR reports was higher when ADR reports had multiple suspected drugs. Although polypharmacy might reflect a severe disease that requires the use of multiple drugs, a previous assessment of the severity of the reported ADRs found that multiple drug exposure might more often lead to serious ADR reports compared to single drug use [13]. Another study found that the use of more than four drugs simultaneously positively correlated with ADR occurrence [14]. Polypharmacy increases the chance of drug-drug interactions and the possibility of ADR occurrence [15, 16]. Because our finding was based on the examination of spontaneous reports, we simply observed reporting tendency. However, considering previous findings in addition to our own, we may pay particular attention to ADRs for children who are prescribed two or more drugs to minimize the risk of serious ADRs.

This study showed that there are many ADR reports associated with immunosuppressants. Because the JADER is a database comprising serious ADR reports, it might contain a lot of information about drugs that are 
Table 3 Ten most frequently reported reactions according to age group

\begin{tabular}{|c|c|}
\hline \multicolumn{2}{|l|}{ a. $<10$ years $(n=18,022)$} \\
\hline Seizure, n (\%) & $\begin{array}{l}390 \\
(2.2)\end{array}$ \\
\hline Anaphylactic reaction, n (\%) & $\begin{array}{l}374 \\
(2.1)\end{array}$ \\
\hline Abnormal behavior, n (\%) & $\begin{array}{l}374 \\
(2.1)\end{array}$ \\
\hline Hepatic function abnormal, n (\%) & $\begin{array}{l}336 \\
(1.9)\end{array}$ \\
\hline Pyrexia, n (\%) & $\begin{array}{l}319 \\
(1.8)\end{array}$ \\
\hline Anaphylactic shock, n (\%) & $\begin{array}{l}271 \\
(1.5)\end{array}$ \\
\hline Stevens-Johnson syndrome, n (\%) & $\begin{array}{l}246 \\
(1.4)\end{array}$ \\
\hline Rash, n (\%) & $\begin{array}{l}229 \\
(1.3)\end{array}$ \\
\hline Erythema multiforme, n (\%) & $\begin{array}{l}195 \\
(1.1)\end{array}$ \\
\hline Drug eruption, n (\%) & $\begin{array}{l}156 \\
(0.9)\end{array}$ \\
\hline \multicolumn{2}{|l|}{ b. $10-19$ years $(n=15,157)$} \\
\hline Abnormal behavior, n (\%) & $\begin{array}{l}419 \\
(2.8)\end{array}$ \\
\hline Anaphylactic shock, n (\%) & $\begin{array}{l}353 \\
(2.3)\end{array}$ \\
\hline Anaphylactic reaction, n (\%) & $\begin{array}{l}333 \\
(2.2)\end{array}$ \\
\hline Pyrexia, n (\%) & $\begin{array}{l}236 \\
(1.6)\end{array}$ \\
\hline Seizure, n (\%) & $\begin{array}{l}223 \\
(1.5)\end{array}$ \\
\hline Hepatic function abnormal, n (\%) & $\begin{array}{l}220 \\
(1.5)\end{array}$ \\
\hline Stevens-Johnson syndrome, n (\%) & $\begin{array}{l}175 \\
(1.2)\end{array}$ \\
\hline $\begin{array}{l}\text { Drug reaction with eosinophilia and systemic symptoms, } \mathrm{n} \\
(\%)\end{array}$ & $\begin{array}{l}171 \\
(1.1)\end{array}$ \\
\hline Pancreatitis acute, n (\%) & $\begin{array}{l}170 \\
(1.1)\end{array}$ \\
\hline Rash, n (\%) & $\begin{array}{l}169 \\
(1.1)\end{array}$ \\
\hline
\end{tabular}

Note: The terms are as described in Japanese version 21.0 of MedDRA ${ }^{\odot}$

likely to cause serious ADRs. As for the most frequently reported drugs in Table 2, the number of ADR reports regarding oseltamivir might be increased by the Dear Healthcare Professional Letters. All drugs in the list, safety information regarding the revision of the precautions of package inserts of drugs have been provided in Pharmaceuticals and Medical Devices Safety Information published by Ministry of Health, Labour and Welfare or Drug Safety Update published by the Federation of
Pharmaceutical Manufacturers' Associations of Japan. This information might have boosted the number of ADR reports. Therefore, the list of drugs in Table 2 seemed not to be related to drug use.

The characteristics of ADR reports varied considerably by the pediatric patient age in previous reports [7]. The potential risk of serious adverse events varies with age and the variability in ADRs by pediatric patient age also differs depending on whether children can complain of side effects. In other words, objective reactions may be reported more often by younger children than by older children, and subjective reactions may be reported more often by older children than by younger children. Therefore, information on age is essential in discussions about ADRs, especially in pediatric patients. However, the present study could not obtain age-related information as a continuous variable and it was used as a categorical variable, such as '< 10 years' and ' $10-19$ years', and it was the weakest attribute related to the JADER. In this study, objective reactions were mainly reported. Pediatric ADR reports, therefore, need to be considered with a more detailed age classification. Age information should have been reported as a continuous variable in original ADR reports; however, the JADER only includes age information as a categorical variable because of privacy considerations. To increase the availability and value of the JADER, age information as a continuous variable should be disclosed, especially in pediatric ADR reports.

Many abnormal behaviors related to oseltamivir administration were reported in 2007 and many abnormal behaviors related to zanamivir were reported in 2009 . Abnormal behaviors related to oseltamivir created concern, and the Dear Healthcare Professional Letters about the abnormal behaviors related to oseltamivir were published by a Japanese regulatory agency on November 27, 2007. In addition, the use of zanamivir, a similar drug to oseltamivir, increased with the advent of the oseltamivirresistant virus in 2008-2009. In early post-marketing phase vigilance (EPPV), a unique system of postmarketing surveillance started in October 2001 in Japan, medical representatives regularly visit medical institutions during the first 6 months of marketing to collect ADRs, so a positive association between the EPPV period and the number of ADRs reported has been suggested $[17,18]$. However, EPPV did not have a positive impact on the increase in the number of ADR reports regarding abnormal behavior related to oseltamivir in 2007 because EPPV for oseltamivir was conducted in 2002 in Japan. ADR reports on "oseltamivir and abnormal behavior" and "zanamivir and abnormal behavior" were thought to have become frequent just after the publication of the letters, although the causal relationship between oseltamivir and abnormal behavior has not been clarified. However, because the percentage of 


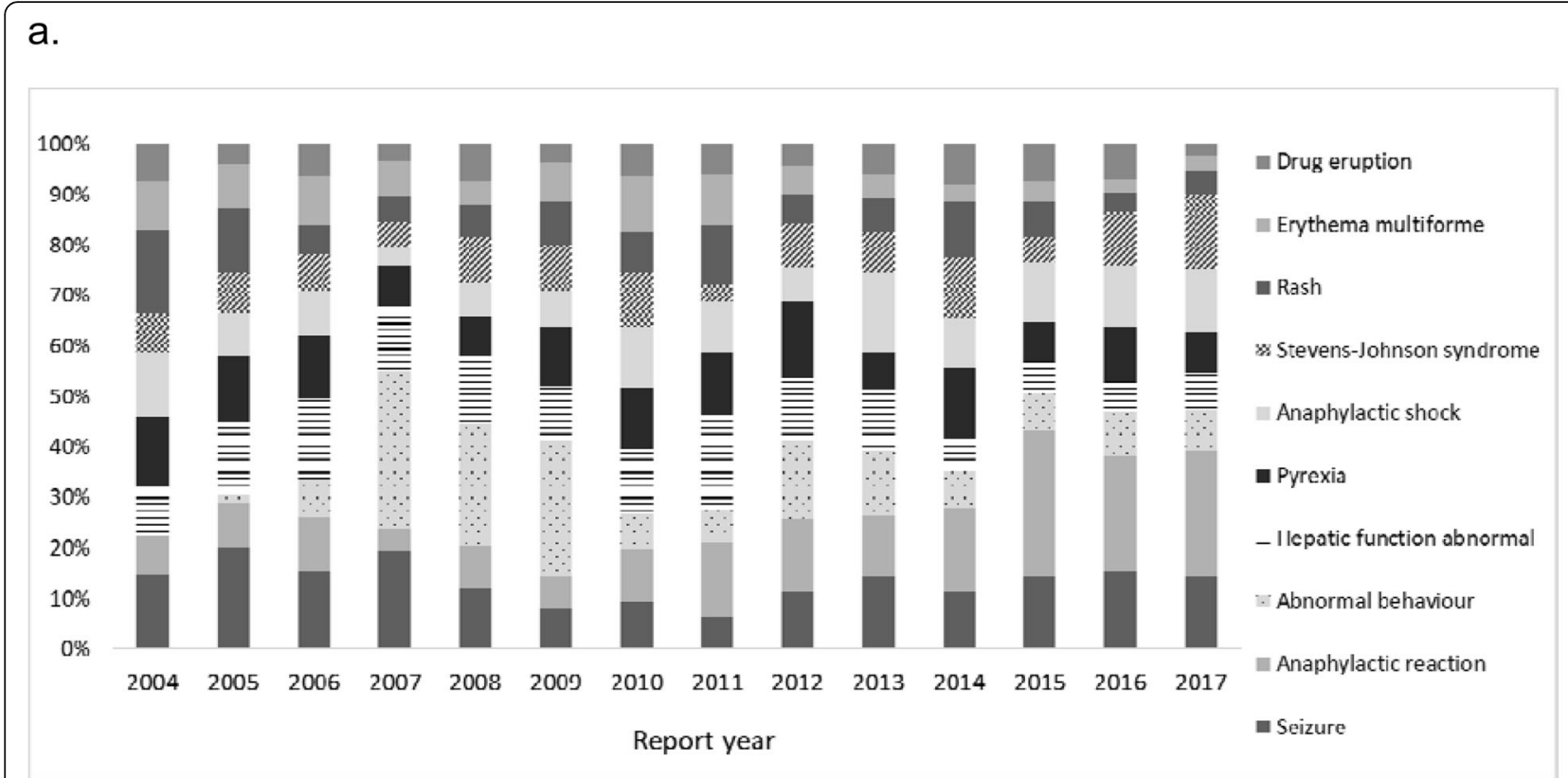

b.

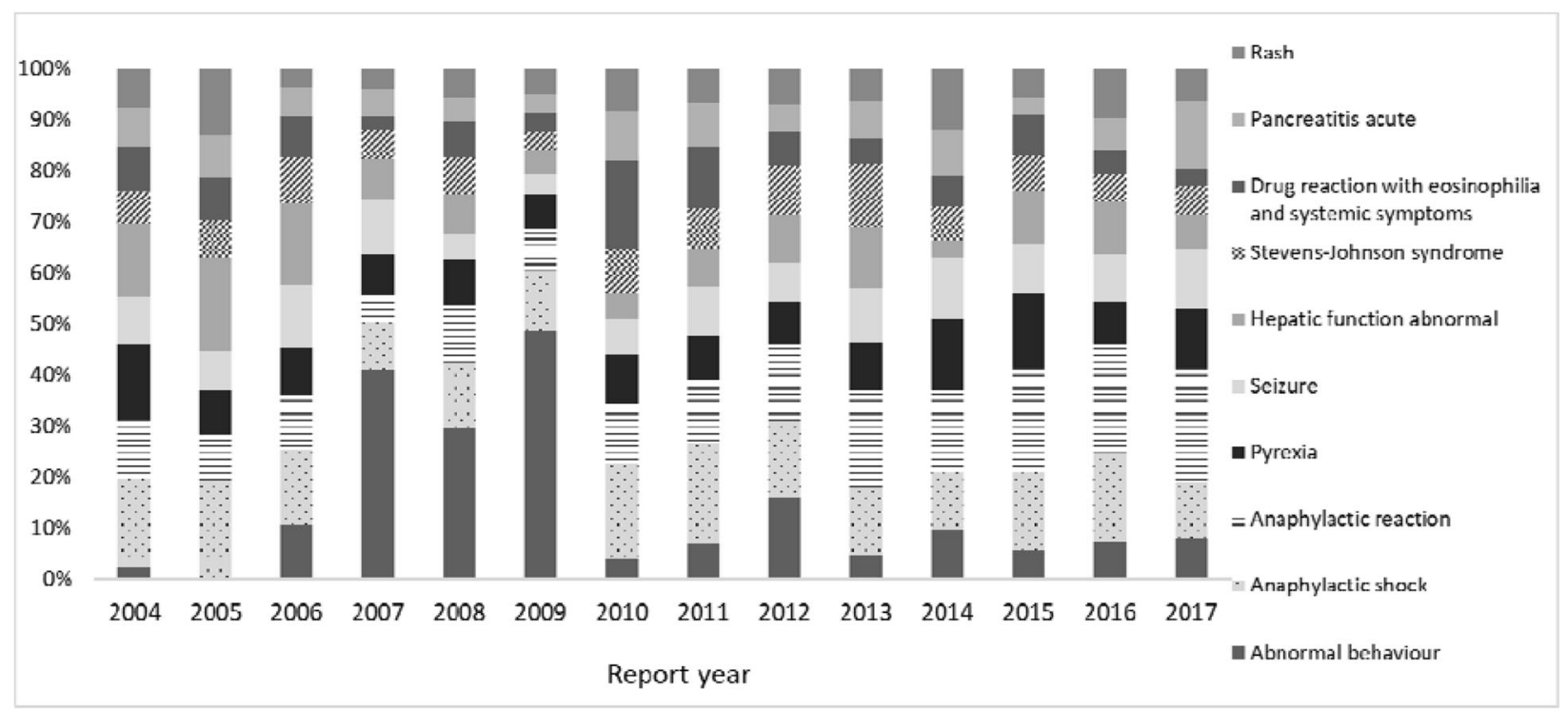

Fig. 3 Time trend for the 10 most frequently reported adverse reactions according to age group. a children aged $<10$ years; $\mathbf{b}$ children aged 10-19years

reports on "anti-influenza virus drugs and abnormal behavior" was not so much $(1.1 \%$ under 10 years old and $1.4 \%$ among children aged 10-19 years), those pairs might not influence on detecting the other signals. In Spain, after the publication of warnings on the use of antidepressants and treatment of attention deficit disorder and hyperactivity linked to the risk of cardiovascular and cerebrovascular disorders in pediatric patients by regulatory agencies, the number of ADR reports regarding cardiovascular and cerebrovascular disorders following the use of antidepressants and treatment of attention deficit disorder and hyperactivity increased [8]. The number of reports of toxic epidermal necrolysis (TEN) and StevensJohnson syndrome (SJS) associated with acetaminophen was also very high. This result might be explained by some reasons as follow; acetaminophen is often used for children, and initiation of acetaminophen treatment occurs in response to fever or ear, nose and throat pain, which might be often the prodromal symptoms of SJS/ TEN and that of an infectious disease such as 
Table 4 Ten most frequent drug-reaction pairs according to age group

\begin{tabular}{|c|c|c|}
\hline Generic name & Reaction & n (\%) \\
\hline \multicolumn{3}{|l|}{ a. $<10$ years } \\
\hline Oseltamivir & Abnormal behavior & $257(0.8)$ \\
\hline $\begin{array}{l}\text { Rurioctocog alfa pegol } \\
\text { (Genetical recombination) }\end{array}$ & Factor VIII inhibition & $102(0.3)$ \\
\hline Zanamivir & Abnormal behavior & $82(0.3)$ \\
\hline Cyclosporine & Posterior reversible encephalopathy syndrome & $71(0.2)$ \\
\hline Theophylline & Seizure & $53(0.2)$ \\
\hline Acetaminophen & Toxic epidermal necrolysis & $52(0.2)$ \\
\hline Theophylline & Encephalopathy & $50(0.2)$ \\
\hline Oseltamivir & Seizure & $48(0.2)$ \\
\hline Acetaminophen & Stevens-Johnson syndrome & $44(0.1)$ \\
\hline Amoxicillin & Erythema multiforme & $44(0.1)$ \\
\hline \multicolumn{3}{|l|}{ b. 10-19 years } \\
\hline Zanamivir & Abnormal behavior & $216(0.8)$ \\
\hline Oseltamivir & Abnormal behavior & $142(0.5)$ \\
\hline Carbamazepine & Drug reaction with eosinophilia and systemic symptoms & $59(0.2)$ \\
\hline Zanamivir & Hallucination & $59(0.2)$ \\
\hline L-Asparaginase & Pancreatitis acute & $46(0.2)$ \\
\hline Cyclosporine & Nephropathy toxic & $42(0.2)$ \\
\hline $\begin{array}{l}\text { Irradiated platelet concentrate, } \\
\text { leukocytes reduced }\end{array}$ & Anaphylactic reaction & $38(0.1)$ \\
\hline Lamotrigine & Rash & $38(0.1)$ \\
\hline Levetiracetam & Epilepsy & $37(0.1)$ \\
\hline Vincristine & Neutropenia & $37(0.1)$ \\
\hline
\end{tabular}

mycoplasma infection or a viral Infections such as influenza accountable for SJS/TEN [19-21].

ADR monitoring based on spontaneous reports in children is an important safety-monitoring activity compared to that in adults because there are few foundations for evaluating the safety of drugs in children. However, the actual causal relationship needs to be continuously verified separately even if many spontaneous reports have observed and regulatory authorities have issued warnings. It should be recognized that the JADER, a spontaneous report database in Japan, also includes such reports that are not clear the causal relationship.

The present study has several limitations. First, the JADER is a passive system, marked by multiple limitations, such as reporting of temporal association, unconfirmed diagnoses, a lack of denominator of users, and unbiased comparison grope [22]. Because of these limitations, it is usually not possible to establish causality between drugs and adverse reactions from JADER reports. Second, it was not possible to analyze the situation according to WHO age group classification such as children aged 5-17 years because the
JADER only included age information as a categorical variable such as children aged $<10$ and $10-19$ years. Nomura et al. have already compared Japanese ADR reports between the FAERS and the JADER [23]. Although the FAERS included non-US data received by drug companies worldwide and it was possible to select Japanese reports with detailed information for age, they clarified that the FAERS and the JADER had different properties. Therefore, in our study, we clarified the characteristics of ADR reports for Japanese children by using the JADER. Third, in the JADER, detailed information on the source of spontaneous ADR reports was not revealed. Therefore, there remains the possibility of duplicated reports, whereby one case might be reported multiple times. This possibility cannot be completely excluded because there are no identifiers for the same case. The identification and elimination of duplicates from an analysis are advantageous and important for the correct interpretation of the data. In future studies, we will evaluate the ability of the JADER for signal detection based on the characteristics of the JADER clarified in this study. 
a.

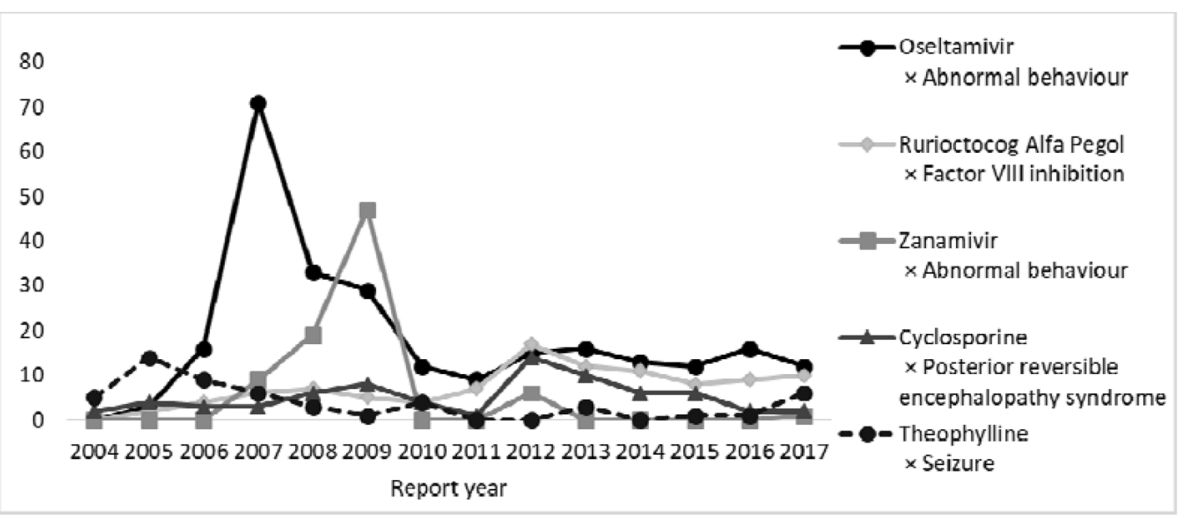

b.

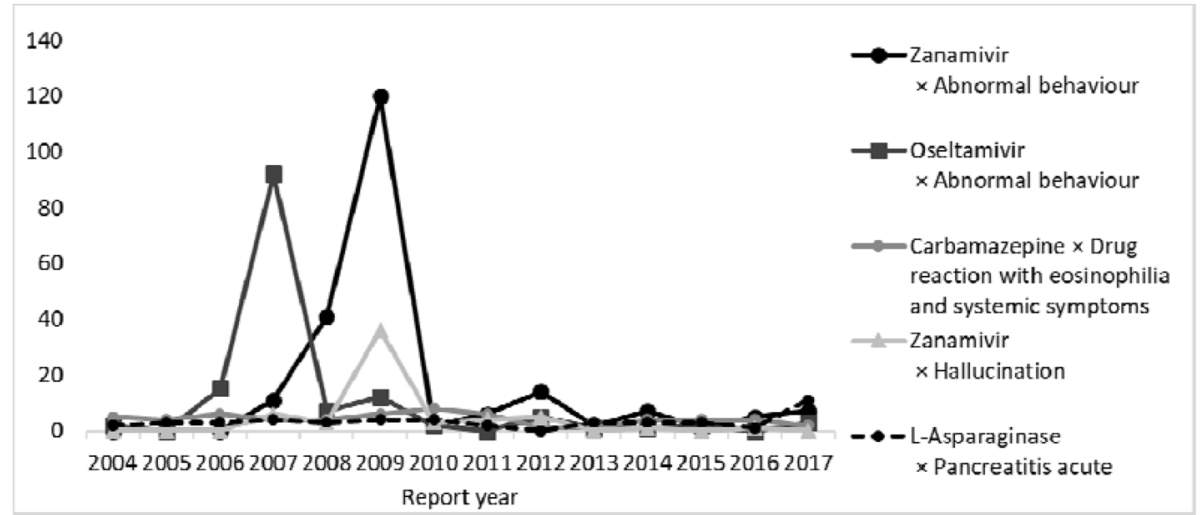

Fig. 4 Time trend for the five most frequently reported drug-reaction pairs according to age group. a children aged $<10$ years; $\mathbf{b}$ children aged $10-19$ years

\section{Conclusion}

We clarified the characteristics of ADR reports for Japanese children by using the JADER. ADR report databases, especially those for pediatric patients, are valuable pharmacovigilance tools in Japan and other countries. Therefore, a proper understanding of the characteristics of the ADR reports in the JADER is important and several limitations such as age group and duplicated reports need to be improved. Additionally, potential signals for ADRs in pediatric patients should be monitored continuously and carefully.

\section{Abbreviations}

ADR: adverse drug reaction; JADER: Japanese Adverse Drug Event Report database; GRiP: Global Research in Pediatrics-Network of Excellence; ICSRs: individual case safety reports; PMDA: Pharmaceutical and Medical Devices Agency; EPPV: early post-marketing phase vigilance; TEN: toxic epidermal necrolysis; SJS: Stevens-Johnson syndrome

\section{Acknowledgements}

The authors would like to thank Rie Suenaga for her technical assistance.

\section{Authors' contributions}

All authors have contributed to this scientific work and approved the final version of the manuscript. AN and TS designed this study, performed the data analyses, and wrote the manuscript. TO was deeply involved in the design of the study and supervised the data analyses. MM, MT, GO, YM, and NM assisted with the data analyses and supervised the drafting of the manuscript. All authors took responsibility for the integrity of the data and accuracy of the data analysis.

\section{Funding}

The design of the study was supported by the grant from the Ministry of Health, Labour and Welfare of Japan (H24-iyakuwakate-011). The

interpretation of data, analysis, and writing the manuscript were supported by the grants from Research on Regulatory Harmonization and Evaluation of Pharmaceuticals, Medical Devices, Regenerative and Cellular Therapy Products, Gene Therapy Products, and Cosmetics from the Japan Agency for Medical Research and Development, AMED (17mk0101095h0001,

$18 \mathrm{mk0101095h0002)}$ and the Japan Society for the Promotion of Science (JSPS) (19 K07213).

Availability of data and materials

The datasets used and/or analyzed during the current study are available from the corresponding author on reasonable request.

Ethics approval and consent to participate

Ethical approval for the study was obtained from the Institutional Review Board of Tohoku University School of Medicine (2017-1-506). No 
administrative permissions or licenses were required to access the data we used in our study because the data was available for free download from the PMDA website. No consent to participate was required due to the retrospective nature of this study.

\section{Consent for publication}

Not applicable.

\section{Competing interests}

Makoto Miyazaki is an employee of Merck Sharp \& Dohme Corp., a subsidiary of Merck \& Co., Inc., Kenilworth, NJ, USA. Makoto Miyazaki is a graduate student at Tohoku University and has contributed to the present study independently of Merck Sharp \& Dohme Corp., a subsidiary of Merck \& Co., Inc., Kenilworth, NJ, USA.

\section{Author details}

${ }^{1}$ Division of Preventive Medicine and Epidemiology, Tohoku University Tohoku Medical Megabank Organization, Sendai, Miyagi, Japan. ${ }^{2}$ Tohoku University Graduate School of Medicine, Sendai, Miyagi, Japan. ${ }^{3}$ Department of Pharmaceutical Sciences, Tohoku University Hospital, Sendai, Miyagi, Japan. ${ }^{4}$ Drug Informatics, Faculty of Pharmacy, Meijo University, Nagoya, Aichi, Japan. ${ }^{5}$ Laboratory of Clinical Pharmacy, Tohoku University Graduate School of Pharmaceutical Sciences, Sendai, Miyagi, Japan. ${ }^{6}$ Department of Pharmacy, Miyagi Cancer Center, Natori, Miyagi, Japan. ${ }^{7}$ Department of Clinical Pharmaceutics, Tohoku Medical and Pharmaceutical University, Sendai, Miyagi, Japan.

Received: 30 November 2019 Accepted: 12 May 2020

Published online: 24 May 2020

\section{References}

1. de Bie S, Ferrajolo C, Straus SM, Verhamme KM, Bonhoeffer J, Wong IC, Sturkenboom MC; GRiP network. Pediatric Drug Safety Surveillance in FDAAERS: A Description of Adverse Events from GRiP Project. PLoS One. 2015; 10(6) e0130399.

2. CORDIS. Global Research in Paediatrics. [cited 2019 Nov 22]. Available from: https://cordis.europa.eu/project/rcn/97619/factsheet/en.

3. Brewer T, Colditz GA. Postmarketing surveillance and adverse drug reactions: current perspectives and future needs. JAMA. 1999;281:824829.

4. Kimland E, Rane A, Ufer M, Panagiotidis G. Paediatric adverse drug reactions reported in Sweden from 1987 to 2001. Pharmacoepidemiol Drug Saf. 2005; 14(7):493-9.

5. Hawcutt DB, Russell NJ, Maqsood H, Kouranloo K, Gomberg S, Waitt C, Sharp A, Riordan A, Turner MA. Spontaneous adverse drug reaction reports for neonates and infants in the UK 2001-2010: content and utility analysis. Br J Clin Pharmacol. 2016;82(6):1601-12.

6. Kaguelidou F, Beau-Salinas F, Jonville-Bera AP, Jacqz-Aigrain E. Neonatal adverse drug reactions: an analysis of reports to the French pharmacovigilance database. Br J Clin Pharmacol. 2016;82(4):1058-68.

7. Rosli R, Ming LC, Abd Aziz N, Manan MM. A retrospective analysis of spontaneous adverse drug reactions reports relating to paediatric patients. PLoS One. 2016;11(6):e0155385.

8. Aldea A, García Sánchez-Colomer M, Fernández Quintana E, Fernández Quintana E, García SM. Paediatric adverse drug reactions reported to the Spanish Pharmacovigilance system from 2004 to 2009. Eur J Clin Pharmacol. 2012;68(9):1329-38.

9. Obebi Cliff-Eribo K, Sammons H, Star K, Ralph Edwards I, Osakwe A Choonara I. Adverse drug reactions in Nigerian children: a retrospective review of reports submitted to the Nigerian Pharmacovigilance Centre from 2005 to 2012. Paediatr Int Child Health. 2016;36(4):300-4.

10. Kobayashi T, Noda A, Obara T, Tsuchiya M, Akasaka K, Yoshida M, Matsuura M, Sato M, Murai Y, Yamaguchi H, Tsuchiya F, Kihira K, Mano N. Knowledge, attitudes, and practice of hospital pharmacists regarding Pharmacovigilance and adverse drug reaction reporting in Japan. Hosp Pharm. (in press).

11. Clarkson A, Choonara I. Surveillance for fatal suspected adverse drug reactions in the UK. Arch Dis Child. 2002;87(6):462-6.

12. Cliff-Eribo KO, Sammons H, Choonara I. Systematic review of paediatric studies of adverse drug reactions from pharmacovigilance databases. Expert Opin Drug Saf. 2016;15(10):1321-8.
13. Li H, Guo XJ, Ye XF, Jiang H, Du WM, Xu JF, Zhang XJ, He J. Adverse drug reactions of spontaneous reports in Shanghai pediatric population. PLoS One. 2014;9(2):e89829.

14. Zopf Y, Rabe C, Neubert A, Hahn EG. Dormann H. Risk factors associated with adverse drug reactions following hospital admission: a prospective analysis of 907 patients in two German university hospitals. Drug Saf 2008; 31(9):789-798.

15. Rashed AN, Wong IC, Cranswick N, Tomlin S, Rascher W, Neubert A. Risk factors associated with adverse drug reactions in hospitalised children: international multicentre study. Eur J Clin Pharmacol. 2012;68(5):801-10.

16. Knopf H, Du Y. Perceived adverse drug reactions among noninstitutionalized children and adolescents in Germany. Br J Clin Pharmacol. 2010;70(3):409-17.

17. Yamada T, Watanabe $Y$, Kusama M, Sugiyama $Y$, Ono S. Factors associated with spontaneous reporting of adverse drug reactions in Japan. Pharmacoepidemiol Drug Saf. 2013;22(5):468-76.

18. Matsuda S, Aoki K, Kawamata T, Kimotsuki T, Kobayashi T, Kuriki H, Nakayama T, Okugawa S, Sugimura Y, Tomita M, Takahashi Y. Bias in spontaneous reporting of adverse drug reactions in Japan. PLoS One. 2015; 10(5):e0126413.

19. Lebrun-Vignes B, Guy C, Jean-Pastor MJ, Gras-Champel V. Zenut M; French network of regional Centres of Pharmacovigilance and the French investigators for adverse skin reactions to drugs. Br J Clin Pharmacol. 2018; 84(2):331-8.

20. Abe J, Umetsu R, Mataki K, Kato Y, Ueda N, Nakayama Y, Hane Y, Matsui T, Hatahira H, Sasaoka S, Motooka Y, Hara H, Kato Z, Kinosada Y, Inagaki N, Nakamura M. Analysis of Stevens-Johnson syndrome and toxic epidermal necrolysis using the Japanese adverse drug event report database. J Pharm Health Care Sci. 2016;2:14.

21. Ban GY, Ahn SJ, Yoo HS, Park HS, Ye YM. Stevens-Johnson syndrome and toxic epidermal Necrolysis associated with acetaminophen use during viral infections. Immune Netw. 2016;16(4):256-60.

22. Zhou W, Pool V, Iskander JK, English-Bullard R, Ball R, Wise RP, Haber P, Pless RP, Mootrey G, Ellenberg SS, Braun MM, Chen RT. Surveillance for safety after immunization: vaccine adverse event reporting system (VAERS)--United States, 1991-2001. MMWR Surveill Summ. 2003;52(1):1-24.

23. Nomura K, Takahashi K, Hinomura Y, Kawaguchi G, Matsushita Y, Marui H, Anzai T, Hashiguchi M, Mochizuki M. Effect of database profile variation on drug safety assessment: an analysis of spontaneous adverse event reports of Japanese cases. Drug Des Devel Ther. 2015;9:3031-41.

\section{Publisher's Note}

Springer Nature remains neutral with regard to jurisdictional claims in published maps and institutional affiliations.

Ready to submit your research? Choose BMC and benefit from

- fast, convenient online submission

- thorough peer review by experienced researchers in your field

- rapid publication on acceptance

- support for research data, including large and complex data types

- gold Open Access which fosters wider collaboration and increased citations

- maximum visibility for your research: over $100 \mathrm{M}$ website views per year

At BMC, research is always in progress.

Learn more biomedcentral.com/submissions 